\title{
Original
}

\section{Construction of Its Evaluation System in Originally Designed Test-Chamber System and Sporicidal Activity of Aerosolized Hypochlorite Solution to Bacillus subtilis Spores}

\author{
SHU ISHIKAWA ${ }^{1 *}$, SHOHEI UENO', MAI MITSUI ${ }^{1}$, YOSHINOBU MATSUMURA ${ }^{2}$, \\ AND TETSURO HATSUOKA ${ }^{1}$ \\ ${ }^{1}$ Engineering Division, Kajima Corporation, 6-5-11 Akasaka, Minato-ku, Tokyo 107-8348, Japan \\ ${ }^{2}$ Department of Life Science \& Biotechnology, Kansai University, \\ 3-3-35 Yamate-cho, Suita-shi, Osaka 564-8680, Japan
}

Received 31 October, 2017/Accepted 20 August, 2018

\begin{abstract}
Effective spatial disinfection systems are required for human health care, public hygiene, and food and medicine manufacturing. Although some aerosolized disinfectants were already applied to its purpose, accurate evaluation systems were under-constructed. In this study, the spatial sporicidal activity of aerosolized hypochlorite solution (AHS) to dormant cells, Bacillus subtilis spores, was evaluated by an originally designed chamber system. In the test-chamber, AHS was supplied and existed as micro-droplets, and environmental relative humidity (RH) could be controlled. Available chlorine (AC) exposure was also controlled with appropriate AC loading but was influenced by the acidity of AHS. Our results indicated that inactivation of spore was depend on AC exposure amount and time. On the other hand, unsaturated environmental RH markedly decreased spore inactivation. This study indicated that our test-chamber system can provide reproducible test data under a homogeneous air condition, and, thereby, that the data obtained by the chamber system should contribute to predicting the AC-required dose to disinfect a whole building.
\end{abstract}

Key words : Aerosolized hypochlorite solution / Sporicidal activity / Environmental relative humidity / Spatial antimicrobial activity evaluation chamber.

\section{INTRODUCTION}

As strong desire for disinfection and cleanness in a living environment and significance of countermeasures against infectious diseases and food contaminations, disinfection of facilities and sanitary control in living environment become of more importance and effective spatial disinfection or sterilization methods, greatly contributed to them, are expected. To solve these desires, several sanitary control systems, including UV irradiation method and directly air-filtration method, were invented, but it is considered that the gasified disinfectant is most effective even to high resistant

*Corresponding author. Tel: +81-3-5544-0487, Fax: +81-35544-1721, E-mail : ishikaws (a)kajima.com dormant cells like a bacterial spore and has good prospects. Especially, bacterial spores generally are highly resistant to a variety of stresses, including heat, ultraviolet radiation and some disinfectants, and, therefore, spores survived after some kind of antimicrobial treatment against microbial contaminants often cause serious problems in food and pharmaceutical industries. Consequently, there is a need to develop effective sporicidal treatments to reduce hygienic risks (Nakagawa et al., 1998), and gasified sporicidal disinfectants are expected to be commercially available.

Several gaseous disinfectants, such as formaldehyde, ethylene oxide, ozone, hydrogen peroxide vaper and chlorine dioxide, have been reported to possess sporicidal activity and are utilized as an effective spatial disinfecting reagent in food and pharmaceutical industry 
(Rogers et al., 2007; Dusseau et al., 2004; Joslyn, 2001). Gaseous disinfectants are also advantageous for decontaminating atmosphere in large room and building and also for disinfecting device and equipment because of the easy dispersion and diffusional contact on large and complex surface. On the other hand, some gaseous disinfectants do not have enough sporicidal activities at a practical concentration and others have serious toxicity and metal corrosiveness. It is important and necessary to determine the treatment conditions, to monitor their residues and to introduce a ventilation system for keeping safety environment (Rogers et al., 2007). Therefore, the accurate modelling to molecular dispersion of gaseous disinfectant and death-kinetics of microbial cell are required. In the homogeneous disinfectant dispersion condition, it was proposed that the microbial cell death followed the Chick-Watson model (Chick 1908; Watson 1908). Mendes et al. (2007) were also propose that lethal modelling of gaseous disinfectant and its validation under practical conditions was necessary and important to use it in safety and reliability, as its microbicidal activity, like ethylene oxide gas, was extensively affected by environmental conditions.

It is also expected that aerosolized disinfectant solutions, including in aerosolized hypochlorite solution (AHS), are recently interesting for hygiene control of indoor environment in buildings and facility surface, like a gaseous disinfectant (Thorn et al., 2013; Martyny et al., 2005), but few practical uses of AHS were reported because of a shortage of basic experimental data for AHS disinfection. Oh et al. (2005) also suggested that aerosolized disinfectants diffuse in a manner similar to gaseous disinfectants, but it has also been considered experimentally to verify their micro-droplet behaviour (Burfoot et al., 1999). Therefore, it is important to understand the quantitative relationship between the dose of an aerosolized disinfectant and reduction of surviving microorganisms for hygienic control in industrial use. In this research, the test-chamber system, which able to supply aerosolized disinfectant with constant exposure rate, was newly designed and constructed. AHS antimicrobial activities were measured in the chamber under suitable treatment conditions, using Bacillus subtilis spore. Amount of available chlorine (AC) and environmental relative humidity $(\mathrm{RH})$ were influenced to the sporicidal activity of AHS.

\section{MATERIALS AND METHODS}

\section{Bacterial strain and culture condition}

Bacillus subtilis ATCC6633 spore suspension (product code E-MN11, $10^{9}$ spores per $\mathrm{mL}$ ) was purchased from Eiken Chemical Co., Ltd. (Tokyo, Japan). B. subtilis spores were cultivated at $37^{\circ} \mathrm{C}$ without shaking in $\mathrm{NBg}$ medium consisting of $8 \mathrm{~g}$ of Difco ${ }^{\mathrm{TM}}$ nutrient broth (product code 234000, Becton, Dickinson and Company, NJ, USA), $5 \mathrm{~g}$ of $\mathrm{NaCl}$ and $2 \mathrm{~g}$ of glucose per litre $(\mathrm{pH}$ 7.0). NBg agar medium supplementing with $1.5 \%$ agar was also used for colony formation.

\section{Chemicals}

Sodium hypochlorite solution with $>5.0 \%$ AC (product code 197-02206; Wako Pure Chemical Industries, Ltd., Osaka, Japan) was used for aerosolized hypochlorite disinfectant in this study. Sodium hypochlorite pentahydrate with $>39.0 \%$ AC (product code 195-17212; Wako Pure Chemical Ind., Ltd.) was also used as a standard substance for hypochlorite quantification. Weakly acidic (WA) -hypochlorite (pH 6.5) and basic (B) -hypochlorite ( $\mathrm{pH}$ 9.0) solutions were prepared with an addition of $1 \mathrm{~mol} \mathrm{~L}^{-1}$ of $\mathrm{HCl}$ solution. 2- [6- (4'Amino) phenoxy-3H-xanthen-3-on-9-yl] benzoic acid (APF) and 2-[6- (4'-hydroxy) phenoxy-3H-xanthen-3on-9-yl] benzoic acid (HPF) were purchased from Goryo Chemical Inc. (Sapporo, Japan) as a fluorescence probe to detect highly reactive oxygen species (ROS) such as hydroxyl radical and peroxynitrite. APF can also detect hypochlorite ion.

\section{Test-chamber system}

The test-chamber system shown in Fig. 1 was originally designed and constructed for this study (Ishikawa et al., 2016). The chamber was composed of an entrance box, air and AHS supply ports, a discharge port, a thermo-hygrometer, a humidity controller and a pair of gloves attached to a front panel. An HSP-SAN100 ultrasonic mist generator for AHS supply was provided by HSP Co. (Okayama, Japan). Highly purified air (Grade 2, Tomoe Shokai Co., Ltd., Tokyo, Japan) was introduced into the chamber via a filter unit of Millex ${ }^{\circledR}-\mathrm{FH}$ (pore size $0.45 \mu \mathrm{m}$, Millipore Co., MA, USA). A circulating aspirator (WJ-20, Shibata Scientific Technology Ltd., Tokyo, Japan) was connected in the discharge port. A humidity controller composed of TTM-004-2-RAP-TE and TA503BL300V01-EP-BHAN-2M (Toplas Engineering Co., Ltd., Tokyo, Japan) was used to regulate $\mathrm{RH}$ in the chamber with controlling inflow rates of AHS and highly purified air. A thermo-hygrometer (TR-72wf, T\&D Corporation, Nagano, Japan) monitored temperature and $\mathrm{RH}$ during an AHS treatment.

\section{Preparation of spore-attaching (SA) plates}

$B$. subtilis spore was collected with a centrifugation at $4,000 \times g$ for 10 min at $4^{\circ} \mathrm{C}$ in order to replace the buffer and to remove cell debris in the suspension (Eiken Chemical Co., Ltd.), subsequently washed twice with a sterilized distilled water (SDW), and resuspended in SDW. The resultant suspension was diluted stepwise, 


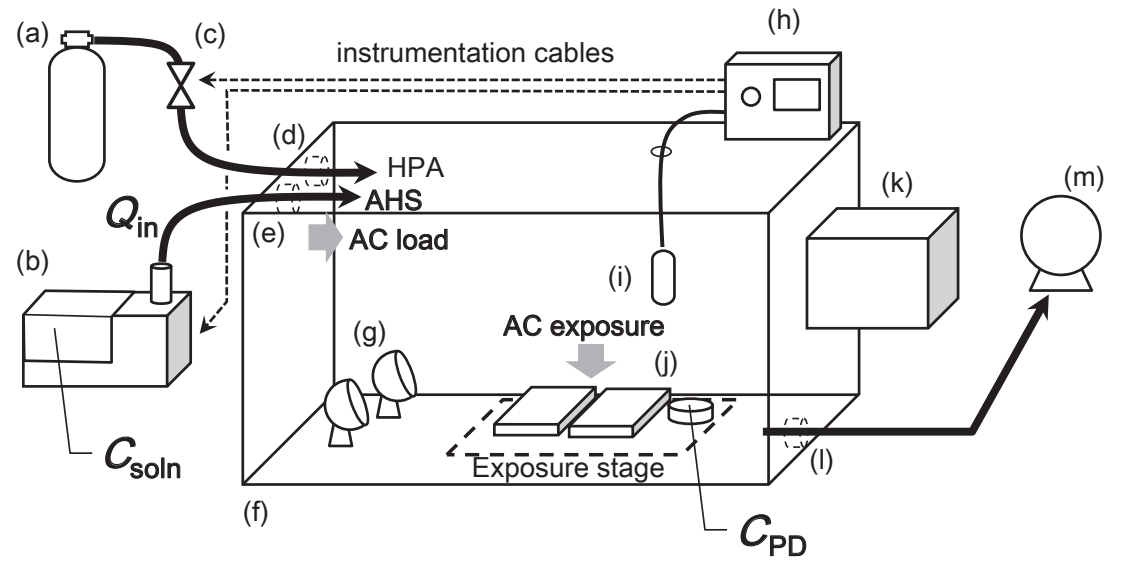

FIG. 1. Schematic diagram of the test-chamber. Arrows indicate the direction of air supply and exhaust. AHS and HPA indicated aerosolized hypochlorite solution and highly purified air, respectively. Each alphabet indicates the components as follows; (a) gas cylinder of HPA, (b) mist generator, (c) solenoid valve, (d) air supply port, (e) AHS supply port, (f) test-chamber (glove box), (g) fan ( $\times 2$ ), (h) humidity controller, (i) thermo-hygro sensor, (j) Spore-attaching (SA) plate (s) and petri dish (s), (k) entrance box, (I) discharge port, (m) aspirator. The parameters which involved to estimate dose of available chlorine (AC) were also indicated as follows; $C_{\text {soln }}, A C$ concentration of the hypochlorite solution in the mist generator; $C_{\mathrm{PD}}, \mathrm{AC}$ concentration in SDW filled in the petri dish; $Q_{\text {in }}$, the supply rate (flow volume) of AHS into the chamber.

and $5-\mu \mathrm{l}$ aliquots of the suspension were subsequently poured onto grids of an inner surface of a lid of a polystyrene 96-well microplate (product code 3870-096; AGC Techno Glass Co., Ltd., Shizuoka, Japan) and dried at room temperature for overnight in a vacuum desiccator. A series of 8 dilutions $\left(10^{6}-10^{-1}\right.$ spores per grid) was spotted in 6 replications (Fig. 2A).

\section{AHS treatment}

WA-hypochlorite and B-hypochlorite solutions adjusted to 9.0-4,000 $\mathrm{mg} \mathrm{L}^{-1}$ of $A C$ were filled into the ultrasonic mist generator. Micro-droplets (less than $10 \mu \mathrm{m}$ average diameter) of AHS generated with it were supplied into the test-chamber at a rate of $73.8 \mathrm{~mL} \mathrm{~h}^{-1}$ and exhausted with an aspiration at $0.72 \mathrm{~m}^{3} \mathrm{~h}^{-1}$ to maintain the spatial $\mathrm{AC}$ concentration at a constant value. $\mathrm{RH}$ in the chamber was kept at saturated condition (95-100\% RH) during the AHS treatment under this condition. As of keeping a $\mathrm{RH}$ of $70 \%$, AHS and high purified air were supplied into the chamber intermittently, and AHS supply rate was, consequently, reduced to $37.6 \mathrm{~mL} \mathrm{~h}^{-1}$. The inner atmosphere of the chamber was stirred using waterresistant fans (SAN ACE 120WS, SANYO Electric Co., Ltd., Osaka, Japan) (Fig. 1), and the air flow in the chamber was also circulated along the inner wall surface. Micro-droplets of AHS were consequently supplied to the SA plates at a horizontal airflow of $0.6 \mathrm{~m} \mathrm{~s}^{-1}$ (Ishikawa et al., 2016).
The SA plates prepared were imported into the chamber through the entrance box and subsequently placed upside down on the exposure stage (Fig. 1 and Fig. 2C). Spores attached on the surface of an SA plate were exposed to AHS at room temperature. At the end of treatment, an SA plate was incubated at room temperature for $1 \mathrm{~h}$ in a dry environment. Finally, the resultant SA plate was mounted on a fresh 96-well microplate fulfilled with a NBg medium (370 $\mu \mathrm{l}$ per well), and cultivated at $37^{\circ} \mathrm{C}$ for $3-4$ days (Fig. 2C). Spore viability on a grid was checked with a turbidity and/or a cell clot in the medium of its corresponding well.

\section{Most probable number (MPN) method for estima- tion of spore survival on an SA plate}

The number of survived (germinable) spores on a grid of an SA plate was estimated (ishikawa et al. 2015) using the most probable number (MPN) method described by Halvorson and Ziegler (1933). The number of survived spores per grid $(N)$ was calculated with the following equation:

$$
N=\operatorname{MPN} \times D=\ln (n / q) \times D
$$

where $D$ is the dilution factor, and $n$ is the number of replicate grids (in this study $n=6$ ) with same initial spore number $\left(10^{6}-10^{-1}\right.$ intact spores in initial), and $q$ is the number of grids showing no growth (no viable spore). 
A

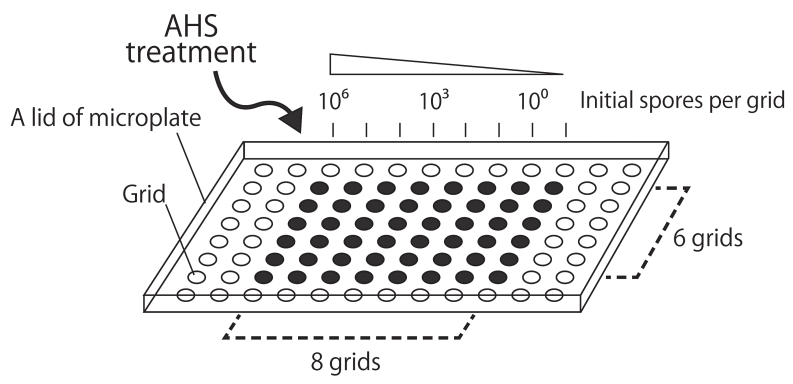

B

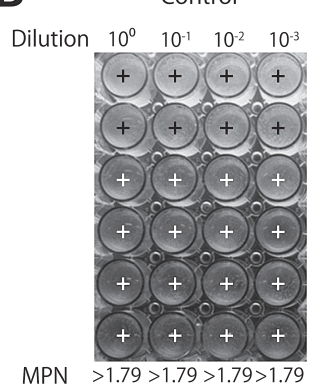

AHS treated

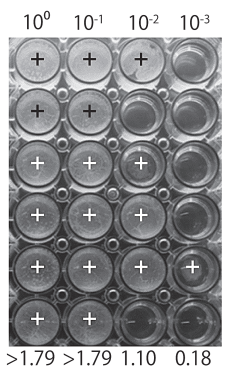

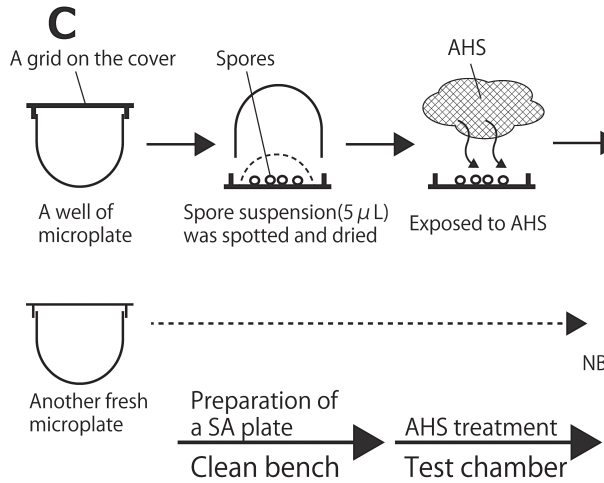

Incubate at RT for $1 \mathrm{~h}$
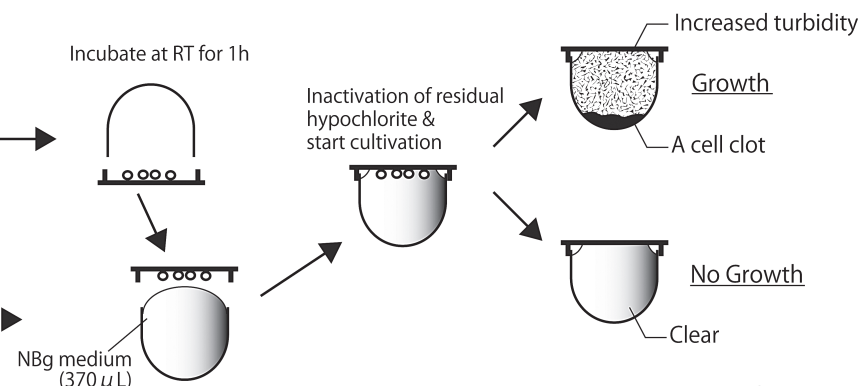
$(370 \mu \mathrm{L})$

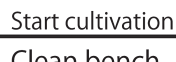

Determination of

the growth

(37 ${ }^{\circ} \mathrm{C}, 3-4$ days)

FIG. 2. Schematics of preparation of a spore-attaching (SA) plate and evaluation of survival spores per grid by most provable number (MPN). Design of SA plate (A), picture of the cultivated SA plate with or without aerosolized hypochlorite solution (AHS) treatment (B), and working scheme of SA plate technique (C) were illustrated. Positive growing grids in (B) were marked with plus $(+)$.

The log spore survival was calculated as the logarithm of the number of surviving spores $(N)$ divided by initial spore number $\left(N_{0}\right)$ :

Log spore survival $=\log _{10}\left(N / N_{0}\right)$

To confirm microbial contamination on an SA plate, a lid of the polystyrene 96-well microplate without spores was also exposed to AHS and cultivated in the same procedure.

\section{Colony count method to detect viable spores}

To validate a viable spore count by the MPN method and an interfering activity of NBg medium to sporicidal action of hypochlorite, survived spores were directly evaluated by colony-forming ability (colony count method). A portion of a spore suspension with or without treated by hypochlorite was diluted with SDW, spread on NBg agar plates, and then cultivated at $37^{\circ} \mathrm{C}$ for $16 \mathrm{~h}$. Colonies were counted as survival spores.

\section{Quantification of AC}

Hypochlorite was quantified by the method described by Setsukinai et al. (2003) using sodium hypochlorite pentahydrate as a standard substance, and the hypochlorite concentrations of a purchased sodium hypochlorite solution and AHS during the treatment were converted to $\mathrm{AC}$ concentration as described by Dychdala (2001). Hypochlorite was evaluated specifically using APF and HPF in this method.

Micro-droplets of AHS during the treatment were captured with a Petri dish (diameter X hight: $34.6 \mathrm{X}$ $17.5 \mathrm{~mm}$ ) filled with $14 \mathrm{~mL}$ of SDW and placed on the exposure stage of the test-chamber. After the AHS treatment, the solution collected in a dish was added to

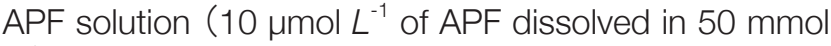
$L^{-1}$ phosphate buffer saline, $\mathrm{pH}$ 7.4) at a volume of $1 / 50$ to 1/10 to measure the amount of ROS and hypochlorite. The resultant cocktail was then incubated at $37^{\circ} \mathrm{C}$ for $1 \mathrm{~h}$. The fluorescence of the reactant was measured using a FP-8200 spectrofluorometer (JASCO Corporation, Tokyo, Japan). The excitation wavelength was $490 \mathrm{~nm}$, and the emission was filtered using a 515-nm barrier filter. The total amount of ROS excluding hypochlorite was quantified using HPF in the same protocol described above.

\section{Evaluation of an AC load and an AC exposure}

The AC Loading rate $\left(\mathrm{mg} \mathrm{m}^{-3} \mathrm{~h}^{-1}\right)$ was calculated as follows.

$$
\text { AC loading rate }=C_{\text {soln }} \times Q_{\text {in }} \times V^{-1}
$$
$C_{\text {soln }}\left(\mathrm{mg} \mathrm{L}^{-1}\right)$ is the AC concentration of the hypochlo- 
A

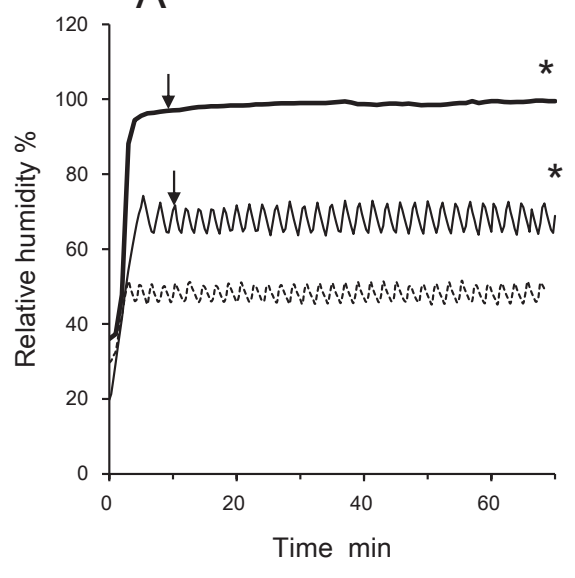

B

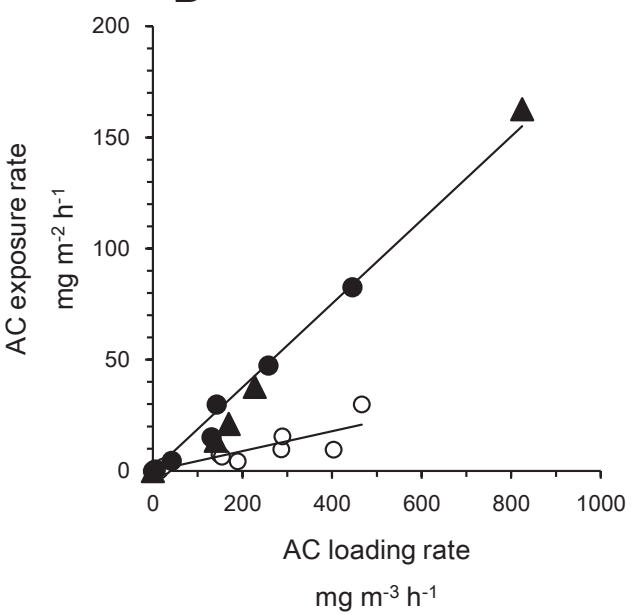

FIG. 3. Aerosolized hypochlorite solution (AHS) treatment condition in the test-chamber system. (A) Relative humidity $(\mathrm{RH})$ in the test-chamber was illustrated with or without a humidity controlling. $\mathrm{RH}$ without a humidity controlling reached to $99.3 \pm 1.0 \%$ (saturated humidity, bold line), and $\mathrm{RH}$ with $70 \%$ and $50 \%$ humidity controlling reached to $68.4 \pm 2.2 \%$ (solid line) and 49.0 $\pm 3.0 \%$ (broken line), respectively. The treatment start time and finish time of the 1-hour AHS treatment were indicated with $(\downarrow)$ and $\left(^{*}\right)$, respectively. (B) Relationship between available chlorine $(A C)$ loading rate introduced into the chamber and $A C$ exposure rate to spore-attaching (SA) plates. The AHS treatment was performed at room temperature. Closed and opened circulars indicated the weakly acidic (WA-, $\mathrm{pH}$ 6.5) and the basic (B-, $\mathrm{pH}$ 9.0) AHS treatment in the saturated humidity, respectively, and triangles indicated the WA-AHS treatment in $70 \%$ humidity.

rite solution set in the ultrasonic mist generator, $Q_{\text {in }}$ is the supply rate $\left(37.6 \mathrm{~mL} \mathrm{~h}^{-1}\right.$ or $73.8 \mathrm{~mL} \mathrm{~h}^{-1}$ ) of AHS introduced into the test-chamber, and $V$ is the volume of the test-chamber $\left(0.18 \mathrm{~m}^{3}\right)$. The AC load $\left(\mathrm{mg} \mathrm{m}^{-3}\right)$ was calculated from the AC loading rate and the treatment time.

The AC exposure $\left(\mathrm{mg} \mathrm{m}^{-2}\right)$, indicated as a practical contact $\mathrm{AC}$ amount onto a target plate, was calculated as follows.

$\mathrm{AC}$ exposure $=$

$C_{\mathrm{PD}} \times($ Volume of SDW filled in a petri dish $) \times$

(Area of petri dish) $^{-1}$

$C_{\mathrm{PD}}\left(\mathrm{mg} \mathrm{L}^{-1}\right)$ is the AC concentration in SDW collected from the petri dish. The AC exposure rate $\left(\mathrm{mg} \mathrm{m}^{-2} \mathrm{~h}^{-1}\right)$ was calculated by division of an AC exposure by a treatment time.

\section{RESULTS}

\section{Performance of the test-chamber system for AHS treatment}

The test-chamber system was originally designed to expose an aerolized disinfectant like AHS to a microorganism contamination surface, such as an SA plate in this study. AHS was able to be kept at a constant concentration under a variety of $\mathrm{RH}$ s in the test-chamber because of continuously and constantly supplying and discharging of AHS micro-droplets. $\mathrm{RH}$ condition in the chamber during an AHS treatment was shown in Fig. 3A. $\mathrm{RH}$ was stable during AHS treatment for 1-3 hour, after the approximate 10-minute transition. $\mathrm{RH}$ at a stable stage were $99.3 \pm 1.0 \%$ (mean \pm standard deviation) during AHS treatment without controlling by the humidity controller (saturated humidity). On the other hand, when the humidity controller was set at $70 \%$ and $50 \% \mathrm{RHs}$, average $\mathrm{RHs}$ were observed at $68.4 \pm 2.2 \%$ and $49.0 \pm 3.0 \%$, respectively. Markedly temperature change was not observed during AHS treatments with a humidity controlling (data not shown).

The relationship between $A C$ loading rate to the chamber and $\mathrm{AC}$ exposure rate onto a target plate was determined, and a corelation was observed between them (Fig. 3B). According to comparison of AC exposure rates, approximately 4.6-fold higher AC exposure rate was observed in WA-AHS treatments, regardless of under saturated humidity or $70 \% \mathrm{RH}$, than in B-AHS treatments under saturated humidity. These results indicated that $\mathrm{AC}$ exposure rate could be controlled with an $\mathrm{AC}$ loading rate and $\mathrm{pH}$ of sodium hypochlorite solution in the mist generator. 


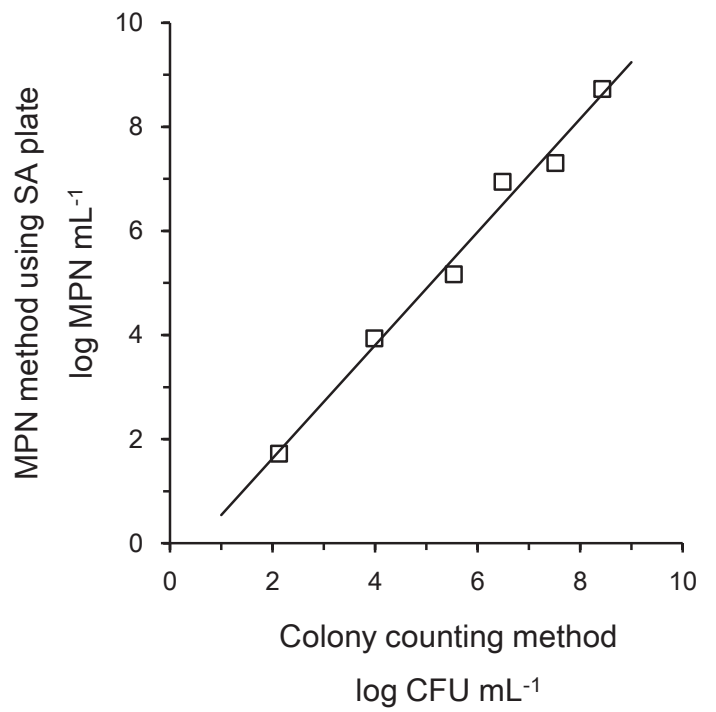

FIG. 4. Comparison of survival spore counts measured by the colony counting method and the MPN method. Each plot was an average value of independent duplicated assays.

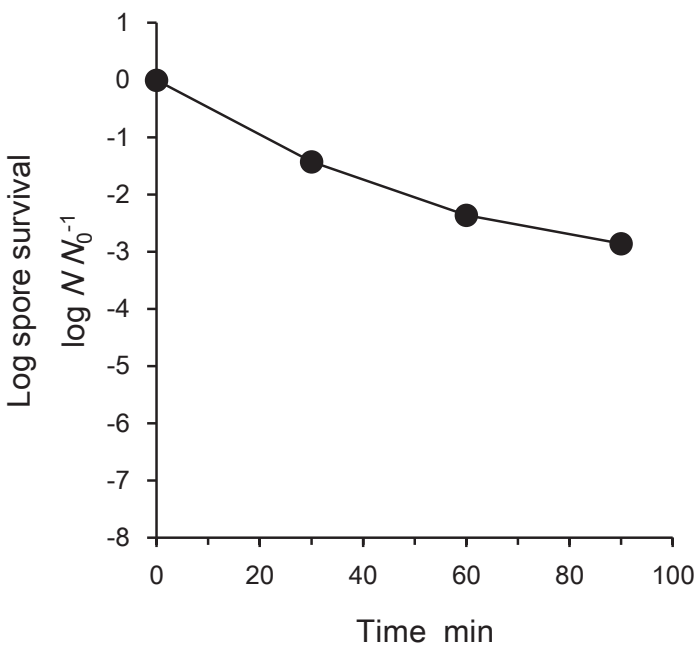

FIG. 5. Reduction of survived spores during the aerosolized hypochlorite solution (AHS) treatment at room temperature. WA-AHS was introduced into the chamber at an available chlorine (AC) loading rate of $15.0 \mathrm{mg} \mathrm{m}^{-3} \mathrm{~h}^{-1}$ under the condition of saturated humidity.

TABLE 1. Inactivation of Bacillus subtilis spores dipped into a weakly acidic (WA-) hypochlorite solution with or without addition of NBg medium. ${ }^{2}$

\begin{tabular}{cccc}
\hline Medium & $\begin{array}{c}\text { Added amount of } \\
\text { WA-hypochlorite } \\
\text { (mg-AC per 1 liter) }\end{array}$ & \begin{tabular}{c} 
Survived Bacillus subtilis spores \\
\cline { 3 - 4 }$\left(\right.$ CFU mL mb $\left.^{-1}\right)$
\end{tabular} & Log reduction ratio \\
\hline SDW & None & $2.1 \times 10^{7}$ & - \\
SDW & 83 & $<1.0 \times 10^{1}$ & $>6.3$ \\
NBg & None & $2.4 \times 10^{7}$ & -0.1 \\
NBg & 83 & $1.9 \times 10^{7}$ & 0.0 \\
\hline
\end{tabular}

${ }^{a}$ Spores were dipped into a WA-hypochlorite solution at room temperature for $1 \mathrm{~h}$.

\section{MPN method using an SA plate was useful to deter- mine number of survival spores}

Comparison of the numbers of viable spores which counted by MPN method with an SA plate and by colony counting method was shown in Fig. 4. In this experiment, intact spores without any disinfection treatments were used. No marked difference was observed between viable spore counts obtained both with these methods. Consequently, this result proposed that estimation of the survived spore number with a MPN method using an SA plate was enough quality for evaluation of disinfection activity in AHS treatment.

\section{NBg medium possessed an interfered activity against the killing reaction of hypochlorite}

The TABLE 1 shows the sporicidal activities of WA-hypochlorite added at $83 \mathrm{mg}$ of AC per 1 liter of $\mathrm{NBg}$ medium and sterilized distilled water. Eighty three milligrams per liter of $\mathrm{AC}$ concentration is approximately
5 times higher than the maximum AC concentration carried over in spore cultivations for MPN method using an SA plate after the AHS treatment.

Spores were suspended in these solutions at a concentration of $2.0 \times 10^{7}$ spores $\mathrm{mL}^{-1}$, and treated for $1 \mathrm{~h}$ at room temperature. Survived spores were evaluated by the colony count method. In present study, the highest AC load in the chamber system was at 824.3 $\mathrm{mg} \mathrm{m}^{-3}$, and concentration of AC carried over in spore cultivation was calculated to $16.9 \mathrm{mg} \mathrm{L}^{-1}$, which is noticeably lower than $83 \mathrm{mg} \mathrm{L}^{-1}$. The ratio of spore inactivation with the hypochlorite treatment in SDW was observed at $>6.0-$ log reduction. No marked decrease of survived spores with the hypochlorite treatment in NBg medium was, however, observed. According to these results, NBg medium had enough inactivation activity for remaining $\mathrm{AC}$ on an SA plate after an AHS treatment. 

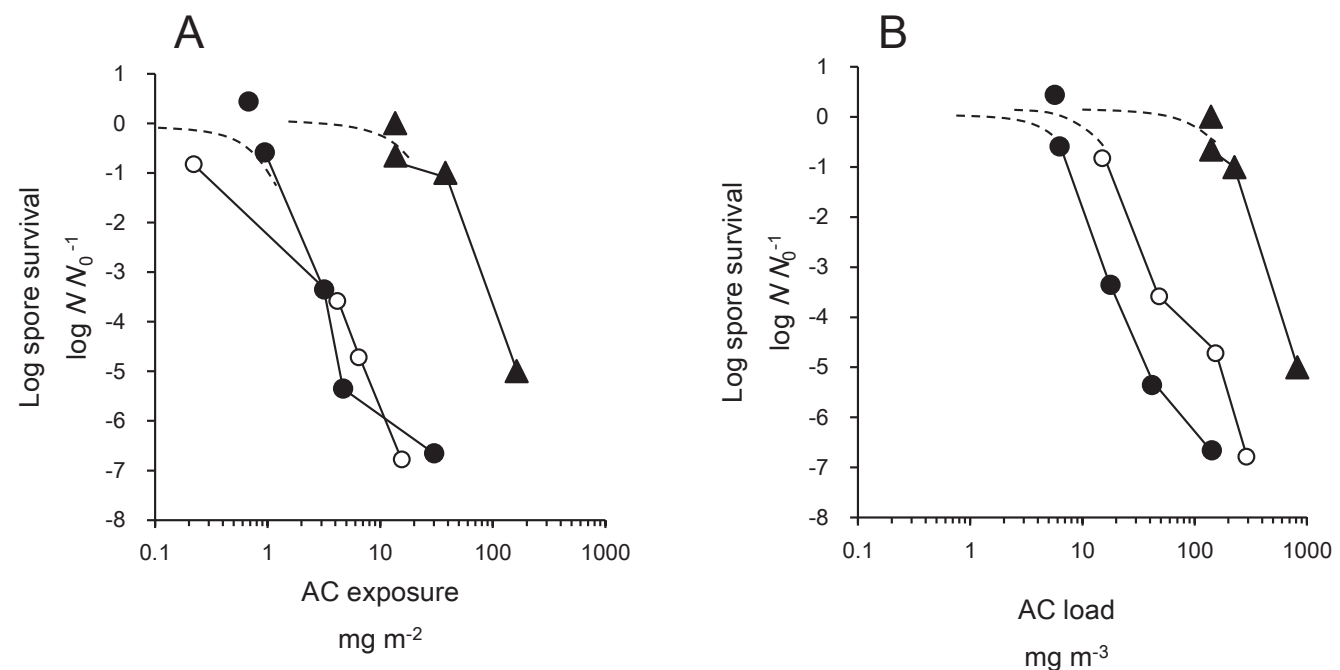

FIG. 6. Log reduction of survival spores attached on a solid surface treated with WA- (closed symbols) and B- (open symbols) aerosolized hypochlorite solution (AHS). Dose in a AHS treatment was presented as available chlorine (AC) exposure on spore-attached (SA) plates (A) and the $\mathrm{AC}$ loads introduced into the chamber (B). RH during the treatment was described saturated humidity (circles) and 70\% RH (triangles). Results were mean values from independent duplicated experiments.

TABLE 2. Required available chlorine (AC) to reduce a survived spore to approximately 5 logs.

\begin{tabular}{|c|c|c|c|c|c|c|}
\hline & & & \multicolumn{4}{|c|}{ Required amount of available chlorine } \\
\hline \multicolumn{2}{|c|}{ AHS treatment } & \multirow{2}{*}{$\begin{array}{l}\text { Log reduction of } \\
\text { survival spores }\end{array}$} & \multicolumn{2}{|c|}{ AC exposure } & \multicolumn{2}{|c|}{ AC load } \\
\hline Hypochlorite solution & $\mathrm{RH}$ & & $\mathrm{mg} \mathrm{m}^{-2}$ & fold & $\mathrm{mg} \mathrm{m}^{-3}$ & fold \\
\hline Weakly acidic (pH 6.5) & Saturated $^{a}$ & 5.4 & 4.7 & 1.0 & 41.7 & 1.0 \\
\hline Basic (pH 9.0) & Saturated $^{a}$ & 4.7 & 6.4 & 1.4 & 153.9 & 3.7 \\
\hline Weakly acidic (pH 6.5) & $70 \% \mathrm{RH}^{b}$ & 5.0 & 162.7 & 34.6 & 824.3 & 19.8 \\
\hline
\end{tabular}

${ }^{a}$ Saturated indicates $99.3 \pm 1.0 \% \mathrm{RH}$.

${ }^{b}$ The measured value of humidity set at $70 \% \mathrm{RH}$ was $68.4 \pm 2.2 \% \mathrm{RH}$ (mean $\pm \mathrm{SD}$ ).

\section{Characterization of the sporicidal activity of the AHS treatment}

The time course in spore survival during WA-AHS treatment under saturated humidity at room temperature was shown in Fig. 5. WA-AHS was introduced into the chamber at an AC loading rate of $15.0 \mathrm{mg} \mathrm{m}^{-3} \mathrm{~h}^{-1}$. Values of the spore survival were decreased gradually as the treatment time increasing.

Relationship between the log spore survival and AC exposure was presented in Fig. 6A. Despite pH difference of $\mathrm{AHS}$, the rate of spore inactivation and $\mathrm{AC}$ exposure were similar between the WA- and B-AHS treatments under saturated humidity (Fig. 6A). Whereas, decreased sporicidal activity in the WA-AHS treatment under $70 \% \mathrm{RH}$ was observed, compared with that under the saturated humidity. The required $A C$ exposure to reduce the spore survival to approximately 5 logs were described in TABLE 2. In 70\% $\mathrm{RH}$, required $\mathrm{AC}$ exposure was observed at $162.7 \mathrm{mg} \mathrm{m}^{-2}$ and this value was approximately 35 -fold higher than those obtained in saturated humidity.

Relationship between the log spore survival and AC loads was also presented in Fig. 6B. Approximately 3.7-fold higher AC load in B-AHS treatment under saturated humidity was required for the similar spore inactivation, compared with in WA-AHS treatment (Fig. 6B and Table 2). This result indicated that $A C$ provided in $\mathrm{B}-\mathrm{AHS}$ treatment was extremely lower than that in WA-AHS treatment. Approximately 20 -fold higher AC load in the WA-AHS treatment under $70 \% \mathrm{RH}$ was required for similar spore inactivation in that under saturated humidity. These results indicated that environmental humidity was also affected to the AHS treatment. 


\section{DISCUSSION}

Hypochlorite solution have been investigated as an aerosolized disinfectant (Thorn et al., 2013; Martyny et al., 2005) because of high microbicidal activity, high safety (low toxicity) for human and easy affordability, especially when its $\mathrm{pH}$ was adjusted to be 5.5-6.5 (Ono et al., 2012). In this study, weakly acidic hypochlorite solution (pH6.5) was used for an aerosolized disinfectant and evaluated its microbicidal activity in our newly designed test-chamber system.

Microbicidal activity of AHS was evaluated by AC exposure and AC load, which were indicated as the amount of AC loaded onto a spore-attaching (SA) plate and the amount of AC loaded into the chamber, respectively. Generally, AC load is easily and exactly controlled during an AHS treatment in practical indoor space, and relationship between $\mathrm{AC}$ exposure and $\mathrm{AC}$ load become useful information. In our study, high correlation between $\mathrm{AC}$ exposure rate and $\mathrm{AC}$ loading rate was confirmed under WA-AHS treatment regardless of relative humidity (Fig. 3B). On the other hand, AC exposure rate in WA-AHS treatment (pH6.5) was markedly higher than those in B-AHS treatment (pH9.0). This result might indicate that $A C$ in micro-droplets of WA-AHS was more retainable than $\mathrm{B}-\mathrm{AHS}$ during the generation of AHS by ultrasonication and/or the AHS treatment. The retentivity of dissolved chlorine in aqueous solution was considered to be high in the following order: $\mathrm{ClO}^{-}>$ $\mathrm{HClO}>\mathrm{Cl}_{2}$ (Fukuzaki 2006; Urano and Fukuzaki 2010), however our results were seemed inconsistent to that. Aerosolizing of hypochlorite solution increased the surface area of micro-droplet so that some material transfer was promoted, and might consequently promoted changes in composition within micro-droplet including volatilization of dissolved chlorine (Urano and Fukuzaki 2010). In other words, aerosolizing methods might be influenced dissolved hypochlorite concentration. However, further detailed investigation is necessary to confirm this hypothesis.

Generally, microbicidal activity of hypochlorite solution was higher under moderately acidic condition than alkaline condition, since the concentration of $\mathrm{HClO}$ with microbicidal activity was increased. It was, therefore, predicted that WA-AHS treatment was more effectives than B-AHS treatment. In fact, however, B-AHS treatment was also effective likewise to WA-AHS on same $\mathrm{AC}$ exposure (Fig. 6A). It was proposed that long-time treatment of B-AHS might wipe the $\mathrm{pH}$ effect of hypochlorite microbicidal action out under the saturated humidity.

On the other hand, although $A C$ exposure rate in WA-AHS treatment at $70 \% \mathrm{RH}$ was equivalent to those in saturated humidity when the $\mathrm{AC}$ loading rate was same (Fig. 3B), the sporicidal activity of the former was remarkably weakened (Fig. 6). Permeability of AC into spores was influenced by or depend on moisture on the SA plate. The effect of moisture on antimicrobial activity of the agents has been also investigated by SantiestebanLópez et al. (2007). Antibacterial susceptibilities of food-borne bacteria to individual and binary mixtures of potassium sorbate with natural phenolic compounds were influenced by $\mathrm{pH}$ and water activity. It was also predicted that lifetime of micro-droplet of AHS in low $\mathrm{RH}$ might be shortened and easily volatilized before attacking spores. Furthermore, if AHS treatment will be planned to achieve the spore reduction of 5 logs under more low humidity conditions, for example $50 \% \mathrm{RH}$, it is necessary to expose AHS with very high AC Load. Such a high AC load must be avoided in our testchamber system, because of safety and damage of the test-chamber. Currently, a new test-chamber system is being designed to carry out AHS treatments under high AC load in safety.

Our study indicated that AHS have enough sporicidal activity under the saturated humidity, but, in fact, it will be difficult to supply micro-droplets of AHS to the entire space of the target room(s) homogeneously, since air quality and airflow in the room are temporally and spatially heterogeneous. Whereas, the test-chamber system used in this study was designed to reproduce an ideal space with a homogeneous air condition. Thereby, the data obtained using the chamber should contribute to predicting the required $\mathrm{AC}$ dose to treat a whole building by integrating the data temporally and spatially.

\section{ACKOWLEDGMENTS}

The authors are grateful to Tomoko Ono, Koji Yamashita and Keiji Kawasaki (HSP Co.) for providing valuable information. They also thank Hiroaki Aikawa, Mariko Ohoi, Masa-aki Hokoyama and Kaori Endoh for supporting the management of this study.

\section{REFERENCES}

Burfoot, D., Hall, K., Brown, K., and Xu, Y. (1999) Fogging for the disinfection of food processing factories and equipment. Trends Food Sci. Technol., 10, 205-210.

Chick, H. (1908) An investigation of the laws of disinfection. J. Hyg., 8, 92-158.

Dusseau, J.-Y., Duroselle, P., and Freney, J. (2004) Gaseous sterilization. In Russell, Hugo and Ayliffe's Principles and Practice of Disinfection, Preservation and Sterilization. 4th edn., (Fraise, A. P., Lambert, P. A., and Maillaed, J.-Y., eds.), pp. 401-435. Blackwell Publishing Ltd, Oxford, UK.

Dychdala, G. R. (2001) Chlorine and chlorine compounds. In Disinfection, Sterilization and Preservation. 5th edn., (Block, S. S., ed.), pp.135-157. Lippincott Williams \& Wilkins, 
Philadelphia, USA.

Fukuzaki, S. (2006) Mechanisms of actions of sodium hypochlorite in cleaning and disinfection process. Biocontrol Sci. 11, 147-157.

Halvorson, H. O., and Ziegler, N. R. (1933) Application of statistics to problems in bacteriology: I. A means of determining bacterial population by the dilution method. J. Bacteriol., 25, 101-121.

Ishikawa, S., Htsuoka, T., Mitsui, M., and Ueno, S. Japanese patent application 2015-188990 (in Japanese). (Sep. 25, 2015).

Ishikawa, S., Htsuoka, T., Ueno, S., Mitsui, M., and Kitta, N. Japanese patent application 2016-62045 (in Japanese). (Mar. 25, 2016).

Joslyn, L. J. (2001) Gaseous chemical sterilization. In Disinfection, Sterilization and Preservation. 5th edn., (Block, S. S., ed.), pp.337-359. Lippincott Williams \& Wilkins, Philadelphia, USA.

Martyny, J. W., Harbeck, R. J., Barker, E. A., Sills, M., Silveira, L., Arbuckle. S., and Newman, L. (2005) Aerosolized sodium hypochlorite inhibits viability and allergenicity of mold on building materials. J. Allergy Clin. Immun., 116, 630-635.

Mendes, G. C. C., Brandão, T. R. S., and Silva, C. L. M. (2007) Ethylene oxide sterilization of medical devices: a review. Am. J. Infect. Control, 35, 574-581.

Nakagawa, K., Shigemoto, M., Matsumura, Y., and Tsuchido, T. (1998) Increased heat resistance of Bacillus subtilis spores in oil. Biocontrol Sci., 3, 87-92.

Oh, S.-W., Gray, P. M., Dougherty, R. H., and Kang, D.-H. (2005) Aerosolization as novel sanitizer delivery system to reduce food-borne pathogens. Lett. Appl. Microbiol., 41,
56-60.

Ono, T., Yamashita, K., Murayama, T., and Sato, T. (2012) Microbicidal effect of weak acid hypochlorous solution on various microorganisms. Biocontrol Sci. 17, 129-133.

Rogers, J. V., Choi, Y. W., Richter, W. R., Rudnicki, D. C., Joseph, D. W., Sabourin, C. L. K., Taylor, M. L., and Chang, J. C. S. (2007) Formaldehyde gas inactivation of Bacillus anthracis, Bacillus subtilis, and Geobacillus stearothermophilus spores on indoor surface materials. J. Appl. Microbiol., 103, 1104-1112.

Setsukinai, K., Urano, Y., Kakinuma, K., Majima, H. J., and Nagano, T. (2003) Development of novel fluorescence probes that can reliably detect reactive oxygen species and distinguish specific species. J. Biol. Chem., 278, 31703175.

Santiesteban-López, A., Palou, E., and López-Malo, A. (2007) Susceptibility of food-borne bacteria to binary combinations of antimicrobials at selected $a_{w}$ and pH. J. Appl. Microbiol. 102, 486-497.

Thorn, R. M. S., Robinson, G. M., and Reynolds, D. M. (2013) Comparative antimicrobial activities of aerosolized sodium hypochlorite, chlorine dioxide, and electrochemically activated solutions evaluated using a novel standardized assay. Antimicrob. Agents Chemother., 57, 2216-2225.

Urano, H., and Fukuzaki, S. (2010) Bactericidal efficacy of ultrasonic fogging with hypochlorite solutions against Escherichia coli on solid surfaces (in Japanese). Bokin Bobai, 38, 573-580.

Watson, H. E. (1908) A note in the variation of the rate of disinfection with change in the concentration of the disinfectant. J. Hyg., 8, 536-592. 